\title{
Aprender a comunicarse con el paciente y con su entorno
}

\author{
J.J. López-Ibor
}

La comunicación es cada vez más esencial en la práctica clínica. La ética dominante en la actualidad es la de autonomía. Su método básico es el consentimiento informado. En otros tiempos era posible actuar desde una perspectiva paternalista, simplemente guiados por el principio de beneficencia, de buscar lo mejor para el enfermo y el médico era el que sabía lo que era mejor. Hoy en día es necesario informar y, para ello, aprender a comunicarse es esencial.

\section{De la medicina de los órganos a la medicina holística}

La medicina científica moderna ha explotado con gran éxito un modelo nacido hace dos siglos y medio, pero que necesita ser incorporado a una perspectiva más amplia. Tuvo su origen cuando los médicos decidieron aplicar los principios de la ciencia entonces naciente y abandonar teorías tradicionales, entre otras las de la medicina galénica. Los médicos aprendieron a ver, tal y como ha descrito Foucault, y las enfermedades fueron asignadas a órganos y a no a humores como antes. Esto es lo que inaugura el libro de Morgagni (1767) De sedibus et causae morborum per anatomen indagatio: la sede y las causas de las enfermedades están en los órganos que pueden ser investigados con métodos anatómicos. Desde entonces se habla de enfermedades del corazón, o del riñón, o del aparato digestivo o del cerebro. Pero también éste es el origen de las especialidades médicas, como la cardiología, la nefrología o la gastroenterología.
En el caso de las enfermedades mentales, el órgano era, naturalmente, el cerebro (Voltaire, Esquirol, Griesinger). Freud, que procedía de la escuela fisiopatológica de Johannes Müller, concedió a la vida psíquica el carácter de una función. De hecho él quiso hacer una psicopatología de la vida cotidiana, y por eso utilizó términos coloquiales que no fueron siempre respetados por sus traductores. Así, utilizó el término seele (lit. 'alma'), que fue traducido al francés como appareil psychique, lo que viene a decir que, igual que tenemos un aparato genito-urinario o respiratorio, forma parte de nuestra economía vital un aparato psíquico. Pero pronto surgió la noción de que había enfermedades mentales que no eran cerebrales, que eran problemas del comportamiento (locura moral de Prichard) o que el elemento psicológico era esencial en la psiquiatría (Griesinger), de ahí que los psiquiatras hayamos tenido que hacernos expertos en un campo que para el resto de los clínicos muchas veces no es más que 'el arte' de la medicina.

$\mathrm{Al}$ médico acuden enfermos, no órganos, de ahí la necesidad de una medicina humanista o antropológica, también científica y no sólo un arte. Se trata de acercarse al mundo de los valores, y considera que el clínico se enfrenta a situaciones para las que la ciencia natural carece aún de respuesta. Sus fuentes son la antropología, el humanismo (filosofía, historia, literatura, etc.) y el psicoanálisis. Trata del sentido de la enfermedad, se pregunta ¿qué significa caer o estar enfermo?, ¿qué relación hay entre estilos de vivir y modos de enfermar? Pretende superar el dualismo modernista. Si las ciencias naturales buscan la generalidad de la ley, las ciencias espirituales,
Catedrático de Psiquiatría. Director del Instituto de Psiquiatría y Salud Mental. Hospital Clínico San Carlos. Madrid, España. E-mail jli@lopez-ibor.com 
en términos del filósofo Dilthey, buscan la singularidad de la persona. Cada enfermo (y cada médico) es un mundo, porque cada ser humano es diferente e irrepetible. Su instrumento no está diseñado para ver, su instrumento es la relación médico-enfermo. Se trata, en palabras de Marañón, buscar al ser humano en la enfermedad y no sólo la enfermedad en el ser humano.

\section{Medicina basada en los valores}

Las respuestas a la complejidad creciente de las decisiones clínicas son dos: una es para afrontar la cantidad y complejidad de datos y evidencia científicos, es la medicina basada en la evidencia (en los hechos); la segunda respuesta pretende resolver los problemas de la cada vez mayor abundancia de conflictos de valores en la práctica médica; se trata de la medicina basada en los valores (Bill Fulford).

Esta nueva propuesta tiene varios antecedentes en la filosofía y en la medicina llamada humanista. De acuerdo con Max Scheler, hay una intencionalidad esencial en la vida emocional y no sólo en la racional. El conocer permite el acceso al mundo de las esencias, y esto es lo que hace la medicina científica natural y la medicina basada en la evidencia (hechos). En cambio, el sentir emocional proporciona un acceso inmediato al mundo de los valores.

Los valores de los seres humanos son diferentes y legítimamente diferentes, y son también particulares. Reparamos en los valores sólo cuando son diversos o conflictivos y, por lo tanto, problemáticos y, por eso, las discrepancias son inevitables. El progreso científico crea nuevas posibilidades de elegir y aumenta la diversidad de los valores humanos en juego. La primera fuente de información es la perspectiva del enfermo o de los grupos de enfermos, a los que concierne una decisión.

En la medicina basada en los valores las diferencias se resuelven por 'disconsenso' (Fulford, 1998), que es un proceso que apoya la acción efectiva a través del equilibrio de valores legítimamente diferentes. La meta de la medicina basada en valores es aceptar la diversidad, y en ella, las habilidades de comunicación son importantes desde la perspectiva de los enfermos o grupos de enfermos.
Mientras que en la medicina basada en la evidencia las diferencias se resuelven con más hechos o datos, con experimentos cruciales, con una mayor información basada en la evidencia o por consenso entre expertos, los conflictos entre valores se resuelven por un proceso diseñado para fortalecer un equilibrio de perspectivas legítimamente diferentes y no por referencia a una regla que prescribe el resultado correcto.

\section{Cómo descubrir la enfermedad y conocer al enfermo}

El conocimiento consiste en descubrir lo oculto. Todos los que han cultivado la filosofía del conocimiento se han enfrentado al hecho de que la verdad debe ser descubierta, que no es algo evidente, que está ahí. En el prólogo del Tratado de Medicina Interna de Harrison aparece una frase, 'La enfermedad descubre sus secretos en paréntesis casuales', que parafrasea un antiguo aforismo de Heráclito: 'La naturaleza esconde celosamente sus secretos'. El conocimiento consiste en hacer visible lo invisible, se trata de aprender a ver, que fue el lema de Leonardo da Vinci a lo largo de su vida. Esto es lo que propugna el método anatomoclínico que, con el enriquecimiento del progreso científico, es la base del quehacer en la práctica cotidiana.

Heidegger, que en su definición de acercamiento a la verdad dice que hay un concepto de verdad, que es el aristotélico tradicional, la adecuación del pensamiento a la cosa. Pero también dice que hay otra que es más antigua, más primitiva que está en la filosofía presocrática, que es el concepto de verdad como aletheia. La palabra aletheia quiere decir revelación, manifestación de las cosas. Hace todo un estudio de cómo se puede uno acercar para que las cosas se revelen en lo que son, y parte de la premisa de que esto es algo que se hace siempre en un diálogo entre personas y en un diálogo basado en la libertad. No se pueden imponer las cosas, porque sino no revelan lo que son, sino que hay que dejarlas que se manifiesten en lo que son.

Este proceso tiene su analogía con la técnica psicoanalítica en la que el analista evita en todo momento inducir cuestiones o respuestas en el analizando. Más abajo veremos cómo todo esto puede llevarse a la práctica clínica cotidiana. 
Karl Popper, en sus Principios para una ética profesional, menciona tres principios para acercarse a la verdad: el de la falibilidad (yo puedo estar equivocado, usted también o lo podemos estar los dos), el del diálogo racional (yo tengo que buscar argumentos racionales, impersonales, en favor y en contra) y el del acercamiento a la verdad con la ayuda del debate. Dice que estos tres principios que son epistemológicos, son a la vez éticos.

\section{La recuperación de la palabra en medicina}

Desde finales del siglo xvin se consolida un proceso de recuperación de la palabra en medicina que culmina en Freud. Comienza por la noción de que guardar secretos, inconfesables, puede ser el origen de una enfermedad. A continuación se expone un ejemplo tomado del libro El descubrimiento del inconsciente de Ellenberger. Se trata de un caso descrito por un médico llamado Aldenhoven:

'Una mujer de 42 años ingresó en el hospital al quinto día de padecer una pneumonía declarada cuando estaba sola en el apartamento sin calefacción. En el momento de su ingreso estaba en situación crítica, exhausta, con gran disnea, cianosis ligera, 120 pulsaciones y temperatura rectal de $40^{\circ} \mathrm{C}$. La radiología mostró una neumonía del lóbulo superior izquierdo. Al día siguiente la situación empeoró, a pesar de la medicación. Aquella misma tarde -la sexta de la enfermedad- el pulso era filiforme, con una frecuencia de 150; aumentó la cianosis y la respiración se hizo muy superficial. La paciente estaba cubierta de sudor frío, la mirada de sus ojos abiertos expresaba angustia y repetía sin cesar que iba a morir. Por la tarde la visitó el Dr. Aldenhoven. En la habitación estaba un viejo amigo de la familia. Aldenhoven ordenó una sangría de 180 centímetros y una inyección de alcanfor. Pensaba que estas medidas prolongarían algo la vida de la paciente, pero que difícilmente detendrían su paulatino declinar; el pulso y la respiración se debilitaban, la mirada ansiosa se oscurecía, la voz era difícilmente perceptible. El médico se sentó en el borde de la cama y dijo a la paciente que su hermana, a la que estaba muy unida, vendría a la mañana siguiente. Ella murmuró débilmente:
Moriré antes... ¡y será el castigo adecuado! ¿Castigo? -preguntó el médico con sosiego-. Bueno, entonces no morirá. Procuraremos que pague la pena sobre la tierra y no debajo.

Estas palabras, que expresaban una convicción médica positiva, dieron en el blanco. La paciente se sintió comprendida. Pidió al visitante que abandonara la habitación y le contó al médico que la pulmonía le había sobrevenido en el lugar en que había sido infiel a su marido (del que estaba alejada y que todavía era prisionero de guerra); ahora, la enfermedad y la muerte representaban el castigo. Inmediatamente después de esta confesión se produjo un cambio extraordinario en el cuadro clínico: se desvaneció la mirada ansiosa de la fisonomía de la paciente, el pulso se hizo más fuerte y lento, la respiración más profunda y tranquila, y la cianosis cedió. Una o dos horas después se había recuperado, hasta el punto de beber alegremente el café de la mañana. El curso posterior de la recuperación se desarrolló sin ningún incidente de interés'.

Sin embargo, es el caso de Anna O., enferma de Breuer, que dio lugar a la publicación de éste y que Freud titulaba Estudios sobre la histeria. Es precisamente la enferma la que introduce el término talking cure, cura mediante la palabra. El resumen de la historia es el siguiente:

'Desde diciembre de 1880 hasta abril de 1881 hubo un período de psicosis manifiesta. En corto plazo aparecieron diversos síntomas: parálisis, contracciones, alteraciones oculares, desorganización lingüística; hablaba una especia de jerga agramatical; su personalidad estaba dividida en una normal, consciente y triste, y otra morbosa, extraña y agitada, que en ocasiones padecía alucinaciones de serpientes negras. Durante ese período, Breuer la visitó con frecuencia; bajo hipnosis ella le contó sus últimos sueños, e inmediatamente se sintió mejorada. Es lo que calificó de cura mediante la charla. En marzo no fue ya capaz de hablar otra lengua que el inglés.

Desde abril hasta diciembre de 1881 los síntomas empeoraron de forma notable. La muerte de su padre el 5 de abril fue un gran choque. No reconocía a nadie, excepto a Breuer, que tuvo que alimentarla durante algún tiempo, y no hablaba sino inglés. Fue trasladada a un sanatorio privado cercano a Viena, donde Breuer la visitaba cada tres o cuatro días. Los síntomas aparecían ahora de forma regular, y fueron aliviados por Breuer mediante sesiones hipnóticas. En lugar 
de contarle sus ensueños, ella le contaba sus últimas alucinaciones.

Desde diciembre de 1881 hasta junio de 1882 la recuperación fue lenta. Las dos personalidades se distinguían ahora perfectamente, y Breuer podía hacerle pasar de una a otra mostrándole una naranja. La característica principal era que la personalidad enferma estaba 365 días más adelantada que la sana. Gracias al diario que había llevado su madre, Breuer pudo comprobar que los acontecimientos que la alucinaban habían ocurrido, día a día, exactamente un año antes. Cierta vez, bajo hipnosis, la enferma contó a Breuer que su dificultad para beber agua databa del día en que vio un perro beber de su vaso. Habiéndole contado esto, el síntoma desapareció. Así comenzó un nuevo tipo de tratamiento: la enferma le narraba a Breuer, en orden cronológico inverso, cada aparición de un síntoma dado, con indicación de la fecha exacta, hasta que alcanzaba la manifestación original y el acontecimiento inicial, y entonces el síntoma desaparecía. Breuer erradicó cada síntoma de esta tediosa manera. Por último, siguió el rastro del último síntoma hasta llegar a un incidente ocurrido mientras cuidaba a su padre enfermo; al sufrir la alucinación de una serpiente negra, se había sentido turbada y había musitado una oración en inglés, la única que le vino a la mente. Tan pronto como recuperó ese recuerdo, la parálisis desapareció de su brazo y pudo hablar alemán. Anna había decidido y anunciado antes que estaría curada para el 7 de junio de 1882, esto es, en el aniversario del día en que entró en el sanatorio a tiempo para las vacaciones de verano. Entonces, según Breuer, abandonó Viena para hacer un viaje, pero todavía tardó cierto tiempo en recuperar su equilibrio completo'.

\section{Transferencia y contratransferencia}

En sus primeros trabajos, Freud observó el desarrollo de una 'intensa relación emocional entre el paciente y el psicoanalista que no se podía explicar por medio de la situación real'. Observó que podía ser 'positiva o negativa y que fluctúa entre el extremo de un amor apasionado por completo sensual y la desenfrenada expresión de desafío y de un odio lleno de amargura'. A este fenómeno lo llamó transferencia; con esta palabra quiso ex- presar la inclinación de los pacientes a dotar a su psicoanalista con muchas características de las personas que primero estuvieron encargadas de su cuidado, en vez de verlas tal y como son. Más adelante comprobó que se trataba de un fenómeno universal de la mente humana que domina la totalidad de las relaciones de cada persona con el entorno. Estos sentimientos se hacen sobre todo patentes cuando la persona enferma siente temores y ansiedad.

Freud percibió también que el subconsciente de los médicos influye también en las relaciones con sus pacientes, lo que denominó contratransferencia.

En concreto, transferencia es la reacción sentimental, en parte consciente, que desarrolla el paciente hacia el médico y en la cual se suelen revivir diversas reacciones de tipo afectivo, experiencias anteriores de amor, hostilidad, dependencia y desconfianza, y otras diferentes según la personalidad del paciente.

Contratransferencia es la reacción simétrica y de signo contrario que elabora el médico hacia el paciente, que puede ser de simpatía, rechazo e inseguridad, entre otras.

\section{Resistencia, represión y conflicto}

Hay mecanismos de defensa involuntarios e inconscientes que impiden que llegue a la mente cualquier material psíquico que pudiese facilitar la emergencia de los contenidos inconscientes reprimidos y que han de ser, muchas veces, superados en la relación médico-enfermo. La noción de resistencia está en la base del psicoanálisis de Freud:

'Había logrado, en efecto, confirmar que los recuerdos olvidados no se habían perdido. Se hallaban a merced del enfermo y dispuestos a surgir por asociación con sus otros recuerdos no olvidados, pero una fuerza indeterminada se lo impedía, obligándolos a permanecer inconscientes. La existencia de esta fuerza era indudable, pues se sentía su actuación al intentar, contrariándola, hacer retornar a la conciencia del enfermo los recuerdos inconscientes. Esta fuerza que mantenía el estado patológico se hacía, pues, notar como una resistencia del enfermo [...]. Las fuerzas que en el tratamiento se oponían, en calidad de resistencia a que lo olvidado se hiciese de nuevo consciente, tenían que ser también las que anteriormente habían producido tal olvido y 
expulsado de la conciencia los sucesos patógenos correspondientes. A este proceso, por mí supuesto, le di el nombre de represión, considerándolo demostrado por la innegable aparición de la resistencia [...]. En todos estos casos se trataba del nacimiento de una optación contraria a los demás deseos del individuo y que, por tanto, resultaba intolerable para las aspiraciones éticas y estéticas de su personalidad. Originábase así un conflicto, una lucha interior, cuyo final era que la representación que aparecía en la conciencia llevando en sí el deseo, inconciliable, sucumbía a la represión, siendo expulsada de la conciencia y olvidada junto con los recuerdos a ella correspondientes. La incompatibilidad de dicha idea con el yo del enfermo era, pues, el motivo de la represión, y las aspiraciones éticas o de otro género del individuo, las fuerzas represoras'.

El reconocimiento del proceso de transferencia, es decir, de la transferencia y de la contratransferencia, es un requisito básico para hacer un buen uso de la relación médico-enfermo, antes de juzgar la respuesta del enfermo, el médico debe preguntarse si su comportamiento o actitud han podido influir en la reacción del paciente.

Es necesario tener presente que la transferencia es un elemento de la relación médicoenfermo que en muchas ocasiones pasa inadvertido. Es frecuente que los enfermos oculten sus miedos, su necesidad de seguridad o nieguen la existencia de esos signos de 'debilidad'. El médico debe prestar atención al desarrollo de la transferencia y de la contratransferencia, de la misma manera que se ocupa de vigilar, por ejemplo, las constantes vitales.

Con el aprendizaje de que la transferencia tiene un papel importante en la actitud emocional del enfermo hacia el médico, éste desarrolla una percepción más realista no sólo del paciente, sino también de sí mismo y de su profesión. Por ejemplo, entendiendo que la actitud agresiva de un paciente puede ser secundaria a la ansiedad provocada por experiencias anteriores, permite al médico tener una visión más amplia del enfermo y de su enfermedad y no interpretarlo como una actitud personal hacia él. La transferencia es importante porque se asocia de manera directa a las posibilidades de curación del enfermo; el médico debe aprender a observar y entender la manera en que el enfermo se relaciona con él, incluso en los casos en los que las señales externas son escasas o pobres.
Diversos estudios han demostrado que una mejor comunicación es una parte esencial para hacer un diagnóstico correcto, mejora el seguimiento del tratamiento, contribuye a la satisfacción del enfermo y mejora la competencia clínica del médico, ya que muchas veces el médico es juzgado en función de su habilidad para comunicarse. En 1992, Jones hizo un estudio en el que demostró que los tutores consideraban como mejor residente a aquel que era capaz de comunicarse mejor y contribuir en el coste económico, una buena comunicación evita la prescripción de fármacos innecesarios y evita errores y denuncias.

\section{Aspectos prácticos: la entrevista clínica}

La entrevista es el instrumento nuclear de la medicina clínica, que tiene tres objetivos básicos: averiguar lo que sucede al enfermo, establecer una relación adecuada entre él y el médico, y proporcionar la información necesaria sobre la enfermedad, su pronóstico y las alternativas de tratamiento.

Para lo primero, el entrevistador deberá obtener información sobre: la enfermedad actual y la biografía del enfermo, realizar las exploraciones pertinentes (que se mencionan más adelante), investigar la dinámica familiar y su capacidad para cuidar del enfermo, y otros datos sobre la situación laboral, familiar y vida social en general, para valorar sus posibles influencias en los padecimientos del enfermo y la repercusión de la enfermedad en ella.

Es necesario aprender a desarrollar la capacidad de escuchar, de establecer una adecuada empatía, de estar atento a la comunicación no verbal durante la entrevista y de identificar las respuestas emocionales del paciente.

Con todo lo anterior se sientan las bases de una relación médico-enfermo adecuada, ya que el enfermo siente el interés del médico por lo que le sucede, capta que el médico es un profesional competente, dispuesto a asumir sus responsabilidades, y aprende a confiar en él.

En tercer lugar, la entrevista sirve para proporcionar información al paciente (informar de cómo prevenir la enfermedad y promover la salud, explicar las consecuencias del diagnóstico, informar de los beneficios del tratamiento y sus posibles efectos secundarios) y, en su caso, a terceros (médicos, familiares, asociaciones que intervienen en el cuidado del paciente). 
Normalmente, el enfermo acude al médico de manera voluntaria con una queja sobre sí mismo, algo está dañado y espera que el médico utilice sus habilidades para identificar lo dañado, qué lo ha producido y que le proporcione alivio.

En la mayoría de las ocasiones, los enfermos acuden acompañados de algún familiar, que lógicamente podrán estar en la entrevista y ser entrevistados junto con el paciente o por separado. En ambos casos, lo ideal es que el enfermo conozca lo tratado y que el médico establezca una relación adecuada con el enfermo y no busque, como en el caso de pronósticos sombríos, alianzas espurias con los allegados (como sería decirle al enfermo que no le pasa nada y al familiar que se trata de un proceso maligno e incurable, con lo cual no sólo no se atiende a la adecuada relación entre el médico y el enfermo, sino que además se establecen barreras de comunicación entre éste y sus familiares abocados a mentirle y disimular o a explicar lo que el médico explicaría mejor).

\section{Anamnesis biográfica}

La anamnesis es, entre otras cosas, una prueba funcional que permite valorar la repercusión de la enfermedad en la vida cotidiana del sujeto. La anamnesis biográfica (Clauser) trata de poner en relación acontecimientos biográficos con síntomas o enfermedades para encontrarles un sentido. Las enfermedades no son nunca hechos casuales.

\section{Técnicas de entrevista}

La entrevista es el fundamento de la relación médico-enfermo; éste se ha de sentir comprendido, que todo lo que dice es tenido en cuenta y jamás deberá sentir que es sometido a un interrogatorio.

La técnica de entrevista se ha enriquecido con aportaciones del psicoanálisis y de la psicología médica en general, donde se reconocen los siguientes aspectos:

- Importancia de la anamnesis.

- Implicación personal del enfermo en su padecimiento.

- Reconocimiento del sentido de los síntomas y de la propia biografía del enfermo.

- Importancia de la relación con el médico mediante la palabra y la transferencia.
- Presencia e implicación personal del médico y su situación, la transferencia y contratransferencia.

Todo entrevistador ha de poner en práctica una serie de cualidades, entre las que destacan: receptividad, tolerancia, atención y confidencialidad.

Toda entrevista ha de tener una estrategia: ¿qué se pretende?, ¿de qué dispongo?, ¿hasta dónde he llegado?, ¿cómo seguir? El entrevistador ha de desarrollar el doble papel de no influir en la entrevista para no contaminar los resultados con sus actitudes y de obtener la máxima información posible, para ello utilizará las técnicas de entrevista no directiva y la anamnesis asociativa, manteniéndose siempre en el más estricto ámbito clínico. La primera entrevista tiene la máxima importancia, ya que es la que ha de obtener la información más significativa y urgente, y definir las reglas de la relación entre el médico y el enfermo. Cuesta un gran esfuerzo enmendar los errores de la primera entrevista.

El médico debe permitir al paciente describir de la manera más eficaz los signos y síntomas, que una vez agrupados, constituyen los diversos síndromes definidos y potencialmente tratables. Para ello es necesario establecer una confianza que permita al paciente hablar con honestidad en un clima de intimidad.

El primer paso es determinar el motivo de consulta, en torno al cual girará el diagnóstico diferencial provisional.

El médico debe dejar que el paciente hable con libertad, emplear una mezcla de preguntas abiertas y cerradas, ahondar en respuestas vagas u oscuras con suficiente persistencia para determinar con precisión la respuesta a una pregunta, descartar las distintas posibilidades diagnósticas mediante preguntas específicas y detalladas, y no temer hacer preguntas relativas a temas que puedan resultar embarazosos o difíciles para el paciente.

Es necesario hacer un uso adecuado de facilitación, empatía, clarificación, confrontación, reafirmación, del silencio y del resumen, y solicitar y reconocer la verbalización de las ideas, preocupaciones, sentimientos del paciente acerca de su enfermedad.

Hay que aprender a evitar los errores que ponen en peligro el desarrollo de una buena entrevista como son: interrumpir innecesariamente al paciente, hacer preguntas largas y complica- 


\section{Tabla. Anamnesis asociativa (Deutsch) o historia psicosomática (Lisansky).}

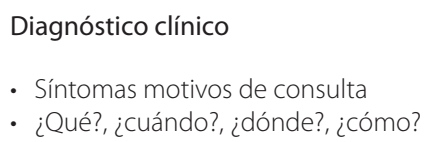

Diagnóstico clínico

- Síntomas motivos de consulta

- ¿Qué?, ¿cuándo?, ¿dónde?, ¿cómo?

Superficie fronteriza

- Sueño, reposo, aficiones, rutina diaria, intereses sociales

Conocer a la persona

- Relaciones intrapersonales e interpersonales sentimientos, afectos, opiniones, valores, temores y esperanzas, actitudes hacia los demás, a sí mismo y el mundo

• ¿Quién?, ¿por qué?

das, utilizar términos técnicos incomprensibles para el enfermo, hacer preguntas de manera que sugieren la respuesta, ignorar la comunicación no verbal, cambiar de tema de repente e inadecuadamente, no preguntar lo suficiente en áreas fundamentales y utilizar un mal estilo (p. ej., dirigirse a un adulto por su nombre de pila o hacerle preguntas demasiado simples, como si de un niño se tratase).

Al terminar hay que dar la oportunidad de preguntar al paciente, y la entrevista debe concluir transmitiendo sensación de confianza y de esperanza, hasta donde sea posible, con la seguridad de que el médico hará todo lo posible por ayudar al enfermo como persona.

La entrevista no directiva fue descrita por Rogers: ésta pretende conseguir la mínima contaminación por parte del médico imponiendo un mínimo intervencionismo, para conseguir que el enfermo exprese lo que realmente siente, experimenta y teme. Pero hay que considerar que toda entrevista se verá limitada por el tiempo. Lo que el médico ha de conseguir es que el enfermo se exprese fluidamente y hacerle recuperar el hilo de la conversación cuando lo ha interrumpido. Saber tolerar y controlar los silencios que tienen lugar cuando afloran a la mente contenidos conflictivos y resistencias necesarias de vencer. Para que el enfermo sea capaz de reanudarlo el médico utilizará una escala progresivamente creciente de incitaciones, gestos, vocalizaciones ('humm...' 'decía...'). Si no son suficientes, podrá repetir la última palabra o frase que dijo el enfermo en un tono neutro. En todo caso evitará la exclamaciones que traducen juicios o prejuicios como ' $\mathrm{iAh}$ claro!' y preguntas que contengan la respuesta o que puedan contestarse con un si o un no ('verdad que el dolor se irradia hacia la nuca').

La anamnesis asociativa fue descrita por Deutsch para ser aplicada a la patología psicosomática y desarrollada por Lisansky con el nombre de historia psicosomática, pretende lograr una relación médico-enfermo auténtica y eficaz, que puede ser captada por el médico y valorada como índice de las reacciones del enfermo a otras confrontaciones personales. La meta de esta entrevista es poder responder a dos cuestiones básicas -qué enfermedad padece y qué tipo de persona es- $y$ tres accesorias -qué factores han contribuido al desarrollo de esa enfermedad, por qué en ese preciso momento, y qué factores incidieron en su evolución y en su respuesta terapéutica.

La técnica parte de distinguir lo que está por encima de la superficie (los síntomas, el motivo de la consulta), del fondo (reacciones interpersonales). Entre ambos se encuentra la superficie (los rendimientos de la vida cotidiana).

El proceso de esta entrevista psicosomática o comprehensiva puede esquematizarse como en la tabla. Los síntomas y motivos están sobre la superficie. Al principio la atención se dirige allí, ya que es el motivo de la consulta. Médico y enfermo intercambian información en torno a preguntas como ¿qué?, ¿cuándo?, ¿cómo? y ¿dónde?

Las relaciones interpersonales e intrapersonales están debajo de la superficie. Aquí se incluye la importantísima información sobre el material psicológico muchas veces reprimido: sentimientos y afectos, opiniones, temores, valores, y actitud frente a sí mismo, los demás y hacia el mundo. Aquí se pregunta ¿quién?, ¿quiénes? y ¿por qué?

La superficie es la frontera, área neutra entre los síntomas y motivos y el material psicológico. Incluye datos como ritmo y modos vitales: sueño, descanso, aficiones, rutina diaria. Han de explorarse en relación con la repercusión funcional de los síntomas.

\section{El proceso de la entrevista}

Los cinco a diez primeros minutos se dedican al motivo de la consulta. Así el médico puede selec- 
cionar otras áreas para continuar la investigación y el enfermo se da cuenta del interés del médico por lo que es evidente. En este corto período de tiempo es posible desarrollar una relación positiva suficiente.

A continuación, en el momento oportuno, el médico plantea algunas cuestiones referentes al área fronteriza, la cual por asociación llevará a temas psicológicos menos manifiestos, que permitiría en caso necesario explorar las relaciones familiares y sociales.

En cualquier momento el médico puede volver a explorar la superficie (síntomas y motivo de la consulta) por varias razones:

- El enfermo expresa de manera impulsiva conflictos psicológicos antes de que el médico sea capaz de discriminar qué información es relevante.

- El material evocado es incómodo para el enfermo o para el médico, lo que amenazaría la continuidad de la entrevista.

- Si el enfermo divaga.

- Al final de la entrevista, a modo de recapitulación.

El área sobre la superficie es el área segura. Es el campo de acción del modelo médico, históricamente consolidado, tolerable desde la perspectiva social del enfermo y con el que el médico ha tenido ocasión de familiarizarse a lo largo de su ejercicio profesional.

El médico no deberá profundizar antes de tiempo, antes de haber establecido una relación adecuada. La atención ha de dirigirse a la comunicación verbal y a la no verbal, para mantener un conocimiento exacto de la situación por encima o por debajo de la frontera. El médico cambia el énfasis de un punto a otro, avanza, retrocede y consolida posiciones para explorar toda el área. Al final, el médico ha de volver al punto inicial, el síntoma o motivo, de manera que quede claro, para permitir un juicio diagnóstico, pronóstico y terapéutico y fundamentar las exploraciones complementarias que se pidan.

El médico deberá tener en cuenta:

- Las palabras del enfermo tienen significados conscientes e inconscientes.

- La comunicación no verbal.

- El lenguaje revela tanto como oculta.

- El enfermo dispone de estrategias de 'diversión', con las que puede o suele estar familiarizado.
- La interferencia existente con los prejuicios, actitudes, irritación, fatiga, propios del enfermo.

- Las proyecciones transferenciales.

- Evitar preguntas dobles o aquellas cuya respuesta esté incluida en su formulación, ya que el enfermo tenderá a repetir las palabras del médico.

\section{Método de la entrevista}

El médico deberá identificar siempre los componentes orgánicos, psicológicos y sociales de las quejas y síntomas. Para ello utilizará preguntas abiertas, que permiten que el individuo se exprese con sus propias palabras.

\section{Aclaración de los síntomas}

Es necesario identificar la naturaleza del síntoma, su intensidad, cualidad, localización, frecuencia, duración, cómo influye en sus relaciones y, por último, el significado que tiene el síntoma para el que lo padece. Todo esto hay que hacerlo para todos y cada uno de los síntomas y quejas que exprese el enfermo.

Cuando el enfermo expresa libremente sus síntomas, el médico es capaz de identificar otras causas o factores que puedan tener una influencia.

\section{Identificación del paciente}

Además de analizar las características del síntoma, es necesario identificar la reacción del paciente cuando se presentan los síntomas. ¿Cuáles son sus fantasías, miedos y ansiedad acerca de los síntomas?, ¿lo ha sentido en otras ocasiones?, ¿conoce a alguien que lo ha padecido? Durante la entrevista se conseguirán datos de su historia clínica anterior, de sus antecedentes personales y familiares. Si el síntoma es identificado como secundario a un acontecimiento vital, o a problemas de tipo social o personal, el médico deberá centrar su atención en identificar el proceso, y lo podrá hacer con el empleo de frases tales como 'no entiendo, podría explicármelo de nuevo o ponerme algún ejemplo', etc.

\section{Aspectos no verbales}

Aunque durante la entrevista la atención se centra en la información que el paciente aporta, en la comunicación verbal es necesario, también, 
tener en cuenta aspectos no verbales de toda comunicación, tales como la entonación que utiliza, los gestos, si es capaz de emitir una sonrisa o si su expresión facial es de tristeza, miedo o cualquier otra emoción.

El aspecto general del paciente puede orientarnos sobre la posible enfermedad de éste y podremos encaminar nuestra entrevista a recoger más datos para hacer un diagnóstico y un diagnóstico diferencial; así, por ejemplo, una cara inexpresiva, de máscara, nos hará sospechar que lo que padece el enfermo es una enfermedad de Parkinson, o una cara de luna llena nos orientará sobre una posible enfermedad de Cushing. También deberemos observar las ropas, la manera de sentarse, sus gestos y ademanes. Si el médico realiza una visita a domicilio, observará su habitación y todo lo que le rodea.

\section{Aspectos terapéuticos de la entrevista}

Los aspectos terapéuticos que se establecen durante la entrevista se deben a la relación que se establece entre el médico y el paciente, y al análisis que hace el médico de la entrevista. Las reacciones de transferencia (del enfermo) y de contratransferencia (del médico) necesitarán ser identificadas. Cuando el médico es capaz de identificarlas, entonces será capaz de dirigir esa orientación para ayudar de la mejor manera al enfermo. Como si de una entrevista de orientación dinámica (psicoterapia) se tratase, el médico empezará a detectar los mecanismos de defensa que el paciente utiliza (negación, resistencia, proyección, identificación, racionalización, sublimación, etc.). Con la apreciación del 'estilo de vida', el médico podrá obtener datos sobre el 'estilo de personalidad' o la manera en que el enfermo se relaciona con los demás y con el ambiente.

La principal función del médico durante el proceso terapéutico de la entrevista es clarificar y objetivar el motivo de consulta. Si tanto el médico como el paciente son capaces de identificar lo que sucede, el tratamiento podrá empezar.

\section{Cómo terminar una entrevista}

Una característica esencial de toda entrevista, independientemente de su duración, es cómo terminarla; una de las mejores maneras es haciendo un resumen, que permitirá al médico ordenar los datos que ha obtenido durante la entrevista; por su parte, el enfermo podrá añadir, quitar o cambiar, o aclarar de nuevo algunos datos. El resumen también permitirá establecer el siguiente paso en la relación médico-enfermo y facilitar el proceso de una entrevista posterior. 